\title{
Journal of Pediatric Neurology \&

\section{Cytokine Storm may Play a Role in the Pathogenesis of Human Parechovirus Type 3-Associated Acute Encephalopathy in Neonates: A Case Report}

Yuichi Abe $^{1^{*}}$, Sanae Machida ${ }^{2}$, Kaori Sassa ${ }^{1}$, Keisuke Okada $^{1}$ and Hideo Yamanouchi ${ }^{1}$

${ }^{1}$ Department of Pediatrics, Saitama Medical University, Japan

${ }^{2}$ Medical Research Center, Saitama Medical University, Japan

"Corresponding author: Yuichi Abe, Department of Pediatrics, Saitama Medical University, Japan, Tel: 81-49-276-1219; Fax: 81-49-276-1219; E-mail: abeyped@saitama-med.ac.jp

Received date: March 17, 2017; Accepted date: April 03, 2017; Published date: April 10, 2017

Copyright: @ 2017 Abe Y, et al. This is an open-access article distributed under the terms of the Creative Commons Attribution License, which permits unrestricted use, distribution, and reproduction in any medium, provided the original author and source are credited.

\begin{abstract}
Background: Human parechovirus type $3(\mathrm{HPeV} 3)$ is an important cause of acute encephalitis in the neonatal and early infantile periods. Typical HPeV3-associated acute encephalitis paradoxically shows no cerebrospinal fluid (CSF) pleocytosis despite virus detection in CSF and diffuse deep white matter involvement of the corpus callosum on magnetic resonance images (MRI).

Patient: An 8-day-old full-term infant was found to have HPeV3 infection of the central nervous system (CNS), which was confirmed using diffusion-weighted MRI showing poor diffusivity in the bilateral deep white matter and the corpus callosum without CNS pleocytosis, as seen in previously reported cases. Interestingly, this patient had extremely elevated serum ferritin and urinary beta-2-microglobulin levels, which indicated a surge of the cytokines tumor necrotizing factor alpha and interferon gamma.
\end{abstract}

Conclusion: These findings suggest that the cytokine storm can promote the pathogenesis of white matter lesions in $\mathrm{HPeV} 3$-associated acute encephalopathy rather than direct infection of the brain.

Keywords: Human parechovirus type 3 (HPeV3); Acute encephalopathy; Ferritin; Beta-2-microglobulin; Cytokine storm

\section{Introduction}

Human parechovirus type $3(\mathrm{HPeV} 3)$ is a common viral agent in all age demographics, but most carriers over 3 months of age develop no severe symptoms beyond a slight cold or gastroenteritis [1]. However, in the new-born and infantile periods before 3 months of age, HPeV3 infection causes severe symptoms, such as sepsis-like syndrome and acute encephalitis [2-4]. Early neonatal and infantile patients with $\mathrm{HPeV} 3$-associated acute encephalitis develop seizures and coma with high fever and the virus is detected from cerebrospinal fluid (CSF), blood, nasopharynx or stool samples [2]. In some previous studies, paradoxically, most neonatal and infantile patients with $\mathrm{HPeV} 3-$ associated acute encephalitis had no CSF pleocytosis despite detection of the virus in CSF and a deep white matter injury as seen on magnetic resonance images (MRI) [2,3,5]. Although no pleocytosis seems controversial to "typical viral encephalitis," the pathogenesis of brain lesions often remains unclear.

Here we report the case of a neonate with acute encephalopathy caused by HPeV3 infection, suggesting that the cytokine storm can affect the pathogenesis of white matter lesions in HPeV3-associated acute encephalopathy rather than direct viral infection of the brain.

\section{Case Report}

An 8 day old new-born male presented with lethargy, fever, and reticular cyanosis and was immediately admitted. The 3,308 g newborn was vaginally delivered at a gestational age of 40 weeks and 2 days with no appreciable complication before or after birth until 2 days after the onset of the presenting symptoms. The parents had no symptoms of infection. The results of initial laboratory examinations indicated no elevation in serum white blood cell count (WBC: 6,500/ $\mu$ l) or Creactive protein level (CRP: $<0.10 \mathrm{mg} / \mathrm{dl})$ and no CSF pleocytosis $(11$ cells/ $\mu \mathrm{l})$. After persistent fever for 2 days from onset, at 10 days of age, brief unilateral hemi convulsions alternately and intermittently occurred on each side for a few hours. Laboratory test results revealed a slight elevation in serum neutrophil-dominant WBC counts $(14,350 / \mu \mathrm{l})$ with neither serum CRP level elevation $(<0.10 \mathrm{mg} / \mathrm{dl})$ nor CSF pleocytosis $(11 \mathrm{cells} / \mu \mathrm{l})$. Meanwhile, initial diffusion-weighted brain MRI showed poor diffusivity in the bilateral deep white matter and the corpus callosum (Figure 1). He was diagnosed as acute encephalitis/encephalopathy andtreated with intravenous administration of $20 \mathrm{mg} / \mathrm{kg} / \mathrm{dose}$ of acyclovir (ACV) three times a day, $30 \mathrm{mg} / \mathrm{kg} /$ day of methylprednisolone (mPSL) one time a day, and 150 $\mathrm{mg} / \mathrm{kg} /$ day ofimmunoglobulin (IVIG) one time a day. Fever disappeared and convulsive seizures were not seen after those therapy. He was discharged on the 11th day of admission. PCR analyses using CSF, serum, pharyngeal mucosal epithelia and stool samples were all positive for HPeV3 [6] but negative for Serumferritin level (1,990 pg/dl at 10 days old of age) and urinary beta-2-microglobulin (B2MG) (4268 $\mu \mathrm{g} / \mathrm{l}$ per $7 \mathrm{mg} / \mathrm{dl}$ urinary creatinine at 8 days of age) were elevated whereas neither serum nor CSF interleukin 6 were elevated $(3.6 \mathrm{pg} / \mathrm{ml}$; normal, $<4.0 \mathrm{pg} / \mathrm{ml}$ and $4.0 \mathrm{pg} / \mathrm{ml}$; normal, $<4.0 \mathrm{pg} / \mathrm{ml}$, respectively). 
Citation: Abe Y, Machida S, Sassa K, Okada K, Yamanouchi H (2017) Cytokine Storm may Play a Role in the Pathogenesis of Human Parechovirus Type 3-Associated Acute Encephalopathy in Neonates: A Case Report. J Pediatr Neurol Med 2: 119. doi: 10.4172/2472-100X.1000119

Page 2 of 3

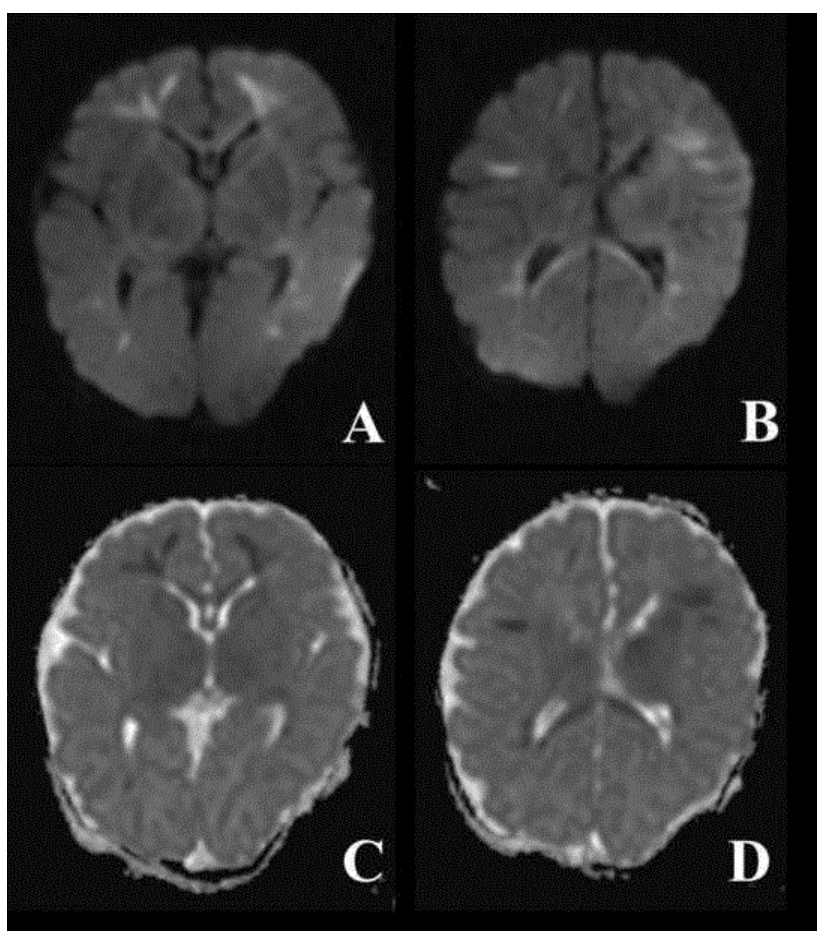

Figure 1: Diffusion-weighted magnetic resonance images $(\mathrm{A}, \mathrm{B})$ and apparent diffusion coefficient maps $(\mathrm{C}, \mathrm{D})$ at 10 days of age. Repetition time $=5400$, Echo time $=160$.

\section{Discussion}

In general, acute viral encephalitis/meningoencephalitis is recognized as a primary viral infection of the brain, such as with herpes simplex virus encephalitis, but is different from that of acute encephalopathy associated with an influenza infection and reveals the following characteristics: pleocytosis in CSF, abnormal findings on MRI, and detection of viral genomes in CSF by molecular biological techniques [7]. Moreover, the diagnosis of direct infection should be technically confirmed by immunopathological methods, if possible [7]. In our patient, however, pleocytosis was not observed despite both detection of $\mathrm{HPeV} 3$ in $\mathrm{CSF}$ and positive lesions seen on MRI. This result indicates that our patient, at least, should not be diagnosed as "typical primary acute encephalitis." A previous report of autopsied neonatal patients with $\mathrm{HPeV} 3$ infection with radiologic evidence of white matter lesions suggested that severe periventricular lesions were pathologically caused by an indirect effect of vascular compromise that is possibly due to viremia because HPeV3 was positive only in the vascular walls and meningeal cells without infection of the parenchymal central nervous system cells even though the virus was detected in CSF without pleocytosis [8]. These findings suggest that many patients diagnosed with $\mathrm{HPeV} 3$-associated encephalitis without pleocytosis also must have no direct infection of the brain parenchyma. According to some previous reports on $\mathrm{HPeV} 3$-associated encephalitis, pleocytosis was hardly observed in most patients with positive lesions seen on MRI despite detection of HPeV3 in CSF $[2,9,10]$. Therefore, we thought that not only our patient but also similar patients with HPeV3-associated acute encephalitis should be diagnosed with acute encephalopathy rather than acute encephalitis.
Moreover, serum ferritin and urinary B2MG levels were elevated in this patient, suggesting hypercytokinemia of tumor necrotizing factor alpha (TNF- $\alpha$ ) and interferon gamma (IFN-r). As well-known results in immunology, excessive production of TNF- $\alpha$ and IFN- $r$ is often estimated by each elevated value of serum ferritin and urinary B2MG, respectively [11-13]. Therefore, measurement of those downstream products of cytokines like serum ferritin and urinary B2MG is meaningful in the clinical setting. A previous report indicated that not only direct infection to brain tissue but also hypercytokinemia is closely related to the pathogenicity of herpes simplex virus encephalitis in neonates [14]. In fact, previously reported neonatal and early infant patients with $\mathrm{HPeV} 3$ infection had elevated serum ferritin, urinary B2MG, and liver enzymes levels and dysfunction of blood coagulation, which are indicative of hypercytokinemia $[11,12,15]$. However, the fine evidence of the relationship between pathophysiology of HPeV3associated encephalitis/encephalopathy and cytokines remains unclear. Volpe speculated that pathological condition of HPeV3 encephalitis may be caused by toll-like receptor (TLR) 8 related to neuronal apoptosis, preoligodendrocyte injury and axonal injury [5]. It is suggested that microglia play a role in creating an environment of oxidative stress through release of inflammatory cytokines, which are causative for white matter injury [16].

If $\mathrm{HPeV} 3$-associated encephalopathy is related to cytokine storm, steroid therapy may be effective. Because our case recovered from a fever within only a day after administration of ACV, MPSL and immunoglobulin, some of those treatments in fact, might have efficacy for the encephalopathy. Especially mPSL has anti-inflammatory effect and is often given to treat with acute encephalopathy caused by cytokine storm [17]. Acute necrotizing encephalopathy (ANE) is famous for an acute encephalopathy with cytokine storm and mainly affects the bilateral thalami, and steroid therapy has a beneficial effect on this type of encephalopathy $[17,18]$. Not only the finding of MRI between HPeV3-associated encephalopathy and ANE, but also a peak age of onset is different from each other $[2,19]$. However, it remains unclear how different and how similar each encephalopathy is caused by cytokine storm.

Collectively, this evidence suggests that hypercytokinemia may be one of the most important elements of the pathophysiology of HPeV3associated acute encephalopathy. On the other hand, both serum and CSF concentrations of interleukin-6 (IL-6) in our patient were not elevated. However, it is possible that the results may be inaccurate because the half-life of IL- 6 is very short and not every sample was immediately frozen. Therefore, more evidence is needed to further elucidate the etiology between HPeV3-associated acute encephalopathy and hypercytokinemia.

\section{Acknowledgement}

This work was supported in large part by a Grant-in-Aid for research (H27-Nanji-Ippan-028) from the Ministry of Health, Labour and Welfare, Japan and also in part by a grant from Japan Agency for Medical Research and Development (40102200).

\section{Author Contribution}

YA wrote the first version of the manuscript. KS and OK were involved in patient management. HD revised the first draft and approved the manuscript. SM detected the virus by experimental methods. 
Citation: Abe Y, Machida S, Sassa K, Okada K, Yamanouchi H (2017) Cytokine Storm may Play a Role in the Pathogenesis of Human Parechovirus Type 3-Associated Acute Encephalopathy in Neonates: A Case Report. J Pediatr Neurol Med 2: 119. doi: 10.4172/2472-100X.1000119

Page 3 of 3

\section{Conflicting Interest}

The authors declared no potential conflicts of interest with respect to the research, authorship, and/or publication of this article.

\section{Research Ethics}

Informed consent about the publication of the clinical information was obtained from the patient's parents.

\section{References}

1. Aizawa Y, Suzuki Y, Watanabe K, Oishi T, Saitoh A (2016) Clinical utility of serum samples for human parechovirus type 3 infection in neonates and young infants: The 2014 epidemic in Japan. J Infect 72: 223-232.

2. Verboon-Maciolek MA, Groenendaal F, Hahn CD, Hellmann J, van Loon AM, et al. (2008) Human parechovirus causes encephalitis with white matter injury in neonates. Ann Neurol 64: 266-273.

3. Renaud C, Harrison CJ (2015) Human parechovirus 3: The most common viral cause of meningoencephalitis in young infants. Infect Dis Clin North Am 29: 415-428.

4. Wolthers KC, Benschop KS, Schinkel J, Molenkamp R, Bergevoet RM, et al. (2008) Human parechoviruses as an important viral cause of sepsis like illness and meningitis in young children. Clin Infect Dis 47: 358-363.

5. Volpe JJ (2008) Neonatal encephalitis and white matter injury: More than just inflammation? Ann Neurol 64: 232-236.

6. Ito M, Yamashita T, Tsuzuki H, Kabashima Y, Hasegawa A, et al. (2010) Detection of human parechoviruses from clinical stool samples in Aichi, Japan. J Clin Microbiol 48: 2683-2688.

7. Chaudhuri A, Kennedy PG (2002) Diagnosis and treatment of viral encephalitis. Postgrad Med J 78: 575-583.

8. Bissel SJ, Auer RN, Chiang CH, Kofler J, Murdoch GH, et al. (2015) Human parechovirus 3 meningitis and fatal leukoencephalopathy. J Neuropathol Exp Neurol 74: 767-777.
9. Gupta S, Fernandez D, Siddiqui A, Tong WC, Pohl K, et al. (2010) Extensive white matter abnormalities associated with neonatal parechovirus (HPeV) infection. Eur J Paediatr Neurol 14: 531-534.

10. Britton PN, Dale RC, Nissen MD, Crawford N, Elliott E, et al. (2016) Parechovirus encephalitis and neurodevelopmental outcomes. Pediatrics 137: e20152848.

11. Miller LL, Miller SC, Torti SV, Tsuji Y, Torti FM (1991) Iron-independent induction of ferritin $\mathrm{H}$ chain by tumor necrosis factor. Proc Natl Acad Sci USA 88: 4946-4950.

12. Heron I, Hokland M, Berg K (1978) Enhanced expression of beta2microglobulin and HLA antigens on human lymphoid cells by interferon. Proc Natl Acad Sci USA 75: 6215-6219.

13. Zandman-Goddard G, Shoenfeld Y (2008) Hyperferritinemia in autoimmunity. Isr Med Assoc J 10: 83-84.

14. Kawada J, Kimura H, Ito Y, Ando Y, Tanaka-Kitajima N, et al. (2004) Evaluation of systemic inflammatory responses in neonates with herpes simplex virus infection. J Infect Dis 190: 494-498.

15. Yuzurihara SS, Ao K, Hara T, Tanaka F, Mori M, et al (2013) Human parechovirus-3 infection in nine neonates and infants presenting symptoms of hemophagocytic lymphohistiocytosis. J Infect Chemother 19: $144-148$.

16. Brownell AD, Reynolds TQ, Livingston B, McCarthy CA (2015) Human parechovirus-3 encephalitis in two neonates: Acute and follow-up magnetic resonance imaging and evaluation of central nervous system markers of inflammation. Pediatr Neurol 52: 245-249.

17. Mizuguchi M, Yamanouchi H, Ichiyama T, Shiomi M (2007) Acute encephalopathy associated with influenza and other viral infections. Acta Neurol Scand Suppl 186: 45-56.

18. Okumura A, Mizuguchi M, Kidokoro H, Tanaka M, Abe S, et al. (2009) Outcome of acute necrotizing encephalopathy in relation to treatment with corticosteroids and gammaglobulin. Brain Dev 31: 221-227.

19. Okumura A, Abe S, Kidokoro H, Mizuguchi M (2009) Acute necrotizing encephalopathy: A comparison between influenza and non-influenza cases. Microbiol Immunol 53: 277-80. 